\title{
Observation of Dirac surface states in the noncentrosymmetric superconductor BiPd
}

\author{
H. M. Benia, ${ }^{1,}$ E. Rampi, ${ }^{2}$ C. Trainer,${ }^{3}$ C. M. Yim, ${ }^{3}$ A. Maldonado, ${ }^{3}$ D. C. Peets,${ }^{1,4}$ A. Stöhr, ${ }^{1}$ U. Starke, ${ }^{1}$ K. \\ Kern, ${ }^{1,5}$ A. Yaresko, ${ }^{1}$ G. Levy, ${ }^{2,6}$ A. Damascelli, ${ }^{2,6}$ C. R. Ast, ${ }^{1}$ A. P. Schnyder, ${ }^{1}$ and P. Wahl ${ }^{3,1},{ }^{1}$ \\ ${ }^{1}$ Max-Planck-Institut für Festkörperforschung, Heisenbergstrasse 1, D-70569 Stuttgart, Germany \\ ${ }^{2}$ Department of Physics \&5 Astronomy, University of British Columbia, Vancouver, British Columbia V6T 1Z1, Canada \\ ${ }^{3}$ SUPA, School of Physics and Astronomy, University of St Andrews, North Haugh, St Andrews, Fife KY16 $9 S S$, UK \\ ${ }^{4}$ Advanced Materials Laboratory, Fudan University, Shanghai 200438, China \\ ${ }^{5}$ Institut de Physique, Ecole Polytechnique Fédérale de Lausanne, 1015 Lausanne, Switzerland \\ ${ }^{6}$ Quantum Matter Institute, University of British Columbia, Vancouver, British Columbia V6T 1Z4, Canada
}

(Dated: September 12, 2016)

\begin{abstract}
Materials with strong spin-orbit coupling (SOC) have in recent years become a subject of intense research due to their potential applications in spintronics and quantum information technology. In particular, in systems which break inversion symmetry, SOC facilitates the Rashba-Dresselhaus effect, leading to a lifting of spin degeneracy in the bulk and intricate spin textures of the Bloch wave functions. Here, by combining angular resolved photoemission (ARPES) and low temperature scanning tunneling microscopy (STM) measurements with relativistic first-principles band structure calculations, we examine the role of SOC in single crystals of noncentrosymmetric BiPd. We report the detection of several Dirac surface states, one of which exhibits an extremely large spin splitting. Unlike the surface states in inversion-symmetric systems, the Dirac surface states of BiPd have completely different properties at opposite faces of the crystal and are not trivially linked by symmetry. The spin-splitting of the surface states exhibits a strong anisotropy by itself, which can be linked to the low in-plane symmetry of the surface termination.

PACS numbers: 79.60.Bm,73.20.-r,71.70.Ej
\end{abstract}

The interplay of strong spin-orbit coupling (SOC) with superconductivity has become a major focus of research in recent years, as both are essential ingredients to stabilize Majorana bound states. The spin-orbit interaction affects the electronic states in a material in various ways and in particular can lead to non-trivial topologies of the band structure. In topological insulators SOC separates the conduction and valence bands, leading to an insulating state with an inverted band gap [1 3]. The latter leads directly to the presence of Dirac surface states protected by time-reversal symmetry [4] 6 . Another consequence of SOC is the Rashba effect 7-10, which in the absence of inversion symmetry lifts the spin degeneracy of the electronic bands, generating intricate spin textures in the electronic wave functions [11 13. Commonly observed at surfaces or interfaces, in noncentrosymmetric materials the Rashba-Dresselhaus effect leads to a lifting of spin-degeneracy of the bulk bands. Combined with superconductivity this can lead to mixing of spin-singlet and spin-triplet pairing components [14, 15] and, more interestingly, to a topologically nontrivial superconducting phase [16 19].

Noncentrosymmetric BiPd [20 24] becomes superconducting below $3.8 \mathrm{~K}$ [25 30] and offers a unique opportunity to study the interplay between SOC and superconductivity. The large spin-orbit interaction of the heavy element Bi results in a sizeable spin splitting of the bulk bands of $\mathrm{BiPd}$ [29]. This in turn can lead to nontrivial wavefunction topologies and unconventional superconducting states [31, 32]. Along with the half-Heusler compounds 33 35] and $\mathrm{PbTaSe}_{2}$ 36 38, BiPd constitutes a rare example of a noncentrosymmetric superconductor which cleaves easily, enabling high-resolution surfacesensitive spectroscopy of its electronic states 29, 39.

In this paper we report the observation of Rashba spinsplit Dirac surface states of noncentrosymmetric BiPd by angle-resolved photoemission spectroscopy (ARPES) and low-temperature scanning tunneling microscopy and spectroscopy (STM/STS). Due to the lack of inversion symmetry, the (010) and (010) surface states can appear at different energies and exhibit different dispersions and spin-polarizations. By combining the experimental results with relativistic first-principles band structure calculations we identify the Dirac surface states of both the (010) and (010) surfaces. This observation of distinct Dirac surface states originating from the opposing surface terminations represents a unique demonstration of the impact of the lack of inversion symmetry on the electronic states.

The crystal growth using a modified BridgmanStockbarger technique has been described in detail elsewhere [40. The crystals were cooled slowly through the $\alpha-\beta$ phase transition to maximize the domain size of the low-temperature $\alpha$ phase; resulting in high-quality crystals [30]. At low temperature $\alpha$-BiPd (in the following referred to as "BiPd") forms in the noncentrosymmetric space group $\mathrm{P} 2_{1}$ [21 24]. The structure is characterized by two double layers stacked along the monoclinic $b$ axis, which are related by a $180^{\circ}$ screw symmetry [see Fig. 1(a)]. Since the bonding between double layers is weaker than within them, the crystals readily cleave perpendicular to the monoclinic $b$ axis and, as previously 

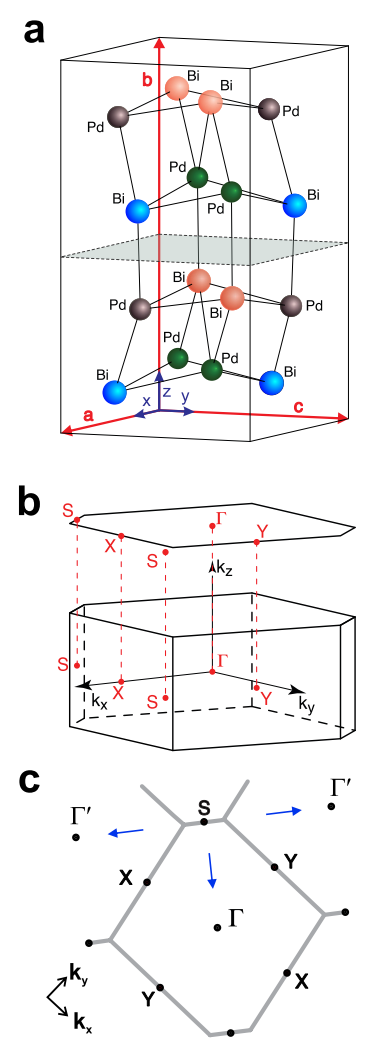
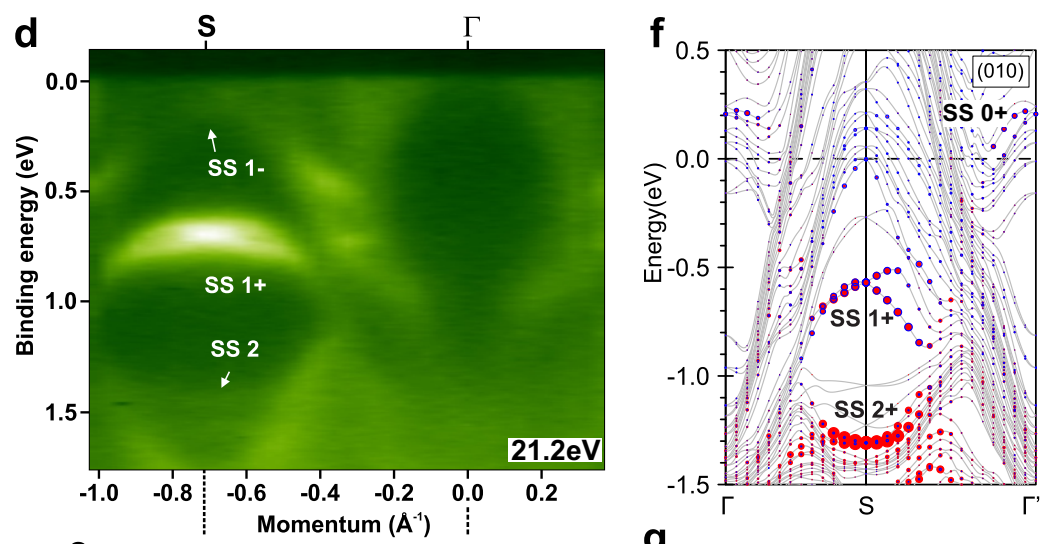

g
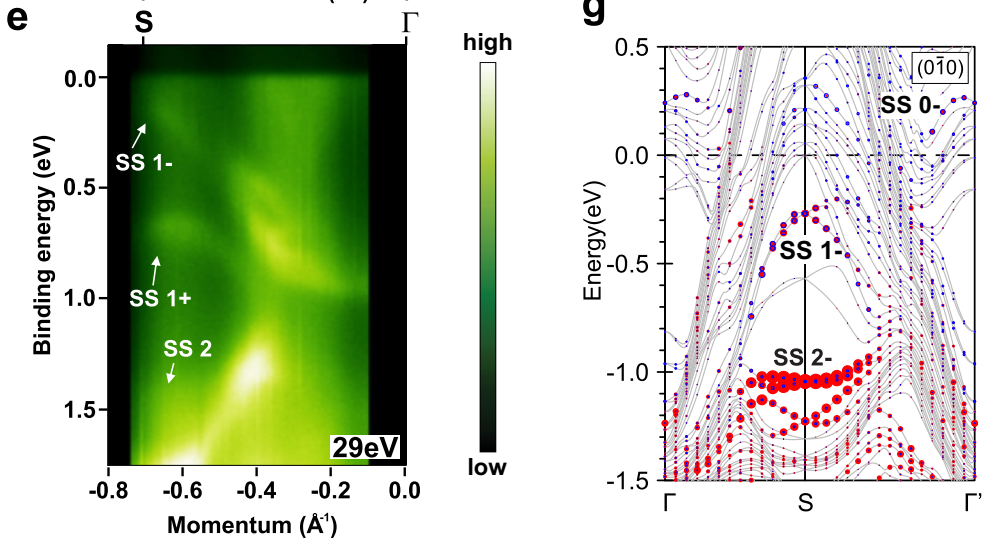

FIG. 1. (a) Crystal structure of BiPd, showing the preferred cleaving plane. (b) Schematic representation of the Brillouin zone of BiPd as well as the surface Brillouin zone of the (010) and (010) surfaces. (c) Surface Brillouin zone with the cuts shown in panels (d)-(g) in blue. (d) Experimental electronic band structure of a $\operatorname{BiPd}(010)$ surface along the $\mathrm{S}-\Gamma$ direction $(\nu=21.2 \mathrm{eV})$. (e) Electronic structure measured with $\nu=29 \mathrm{eV}$. The photoemission intensities in (d) and (e) and in other photoemission intensity maps in this paper are displayed using the colour scale shown in e. (f) and (g) Calculated electronic structure of BiPd in slab geometry including the cuts shown in (d) and (e). The size of the circles is proportional to the spectral weight of Bi $6 p$ states in the first (red) and second (blue) layer of the (010) and (010) surfaces. The surface states are labelled SS $n+$ and SSn-, where + and - denote whether they occur on the $(010)$ or (010) surface, while $n$ numbers the surface states sequentially with increasing binding energy.

demonstrated 29 , are twinned such that both (010) and $(0 \overline{1} 0)$ surfaces can appear on the same side of the crystal (see ref. 41 for details on the cleaving procedure).

ARPES measurements were performed on freshly cleaved surfaces using (i) a Helium source $(\nu=21.2 \mathrm{eV}$ and $40.8 \mathrm{eV}$ ) with a hemispherical SPECS HSA3500 electron analyzer, and (ii) linearly-polarized synchrotron light from the UE112-PGM undulator beamline at BESSY II with a Scienta R8000 analyzer. The sample was held at temperatures lower than $100 \mathrm{~K}$ during cleaving and throughout the measurements.

STM experiments were performed in a home-built lowtemperature STM operating at temperatures down to $1.5 \mathrm{~K}$ in cryogenic vacuum 42 . Samples were prepared by in-situ cleaving at low temperatures. Tips were cut from a PtIr wire. Bias voltages were applied to the sample. Differential conductance spectra have been recorded through a lock-in amplifier $\left(f=408 \mathrm{~Hz}, V_{\text {mod }}=2 \mathrm{mV}\right)$.

Figures 1(b) and (c) show schematically the bulk Bril- louin zone and its surface projection. Figures 1(d) and (e) show the results of ARPES, measured along the $\Gamma-$ $\mathrm{S}$ direction in the Brillouin zone at two different photon energies. The most prominent feature of the surface electronic structure when measured with a He-I lamp is the appearance of a strong state (labelled SS1+ in Fig. 1(d)) at the S-point at $0.7 \mathrm{eV}$ binding energy. In addition, at higher photon energy (Fig. 1(e)), within the same directional band gap at the S-point a surface state SS1- can be identified, albeit with much weaker intensity. These are identified as surface states through their lack of dispersion with varying the incident photon energy and hence $k_{z}$ [41. To understand the origin and topological nature of these surface states, we have employed fully relativistic linear muffin tin orbital calculations 43,45 using a repeated slab system consisting of six BiPd double layers separated by two empty double layers which represent the vacuum. We find that around the Fermi energy $E_{\mathrm{F}}$, all the bands are mainly of $\mathrm{Bi} 6 p$ orbital character with 
subdominant but non-negligible contributions of $\mathrm{Pd} 4 d$ states. The strong atomic $\mathrm{SOC}$ of $\mathrm{Bi}$ induces a spin splitting of the bands of the order of tens of $\mathrm{meV}$ and, moreover, results in a large energy shift of states that have predominant $p_{1 / 2}$ orbital character [46. The latter leads to formation of a band gap at the $\Gamma$ point $[29,46]$. In Figs. 11(f) and (g), we show the calculated dispersions near $E_{\mathrm{F}}$ of the (010) and (010) surfaces of $\mathrm{BiPd}$, respectively, along high symmetry directions of the surface BZ [Fig. 1. (b) and (c)]. The momentum-resolved surface densities of states at the (010) and (010) sides are indicated by filled circles. Interestingly, Dirac surface states appear both at the $S$ and $\Gamma$ points of the surface BZ. Thus by comparison with band structure calculations, the features SS1+ and SS1- seen in ARPES can be directly associated with the surface states of the BiPd surface. The simultaneous observation of SS1+ and SS1- in the measurement is not reproduced in the calculations: the two states originate from opposite surface terminations, with the one at higher binding energy arising from the (010) termination and the one closer to the Fermi energy from the (010) termination. Since these two terminations correspond to opposite surfaces of a single crystal, their simultaneous observation by ARPES indicates twin domains with opposite direction of the crystallographic $b$ axis within the beam spot. A structural transition around $200^{\circ} \mathrm{C}$ [23, 24] is known to cause twinning, and this type of twin boundary has been previously observed by STM 29.

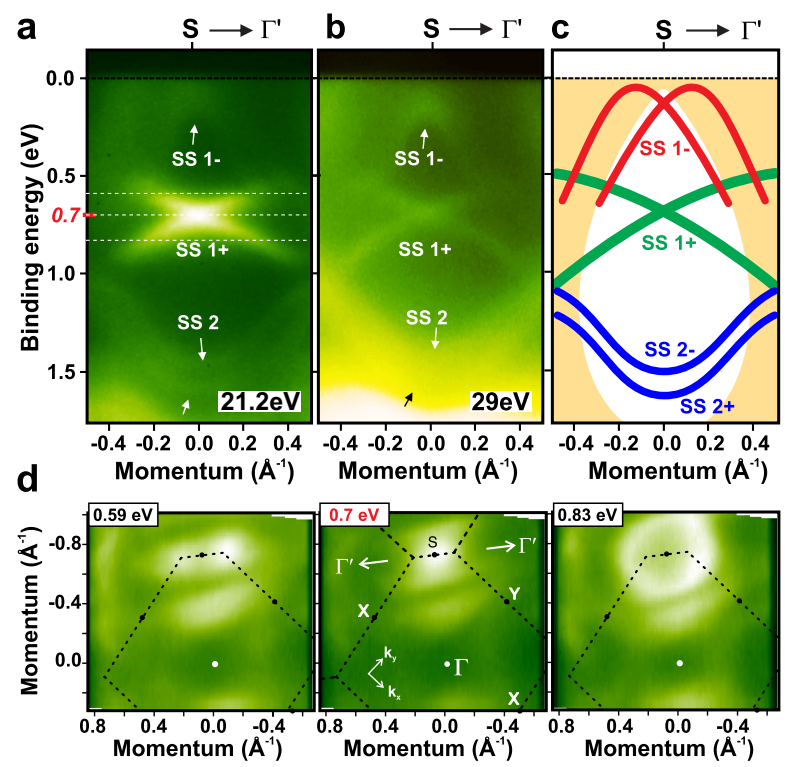

FIG. 2. (a) and (b) Intensity maps of the energy and $k$ resolved surface band structure of $\mathrm{BiPd}$ measured along the $\mathrm{S}-\Gamma^{\prime}$ direction with $\nu=21.2 \mathrm{eV}$ and $\nu=29 \mathrm{eV}$, respectively. (c) Schematic representation of the surface states. (d) Constant energy cuts obtained at $0.59,0.7$, and $0.83 \mathrm{eV}$, energies indicated as dashed horizontal lines in (a). Overlaid on the constant energy cuts is a schematic of the surface Brillouin zone.
In full agreement between experiment and theory, the spin-splitting of the surface state is substantially larger in the $\mathrm{S}-\Gamma^{\prime}$ direction compared to the $\mathrm{S}-\Gamma$ direction. Experimental data for the $\mathrm{S}-\Gamma^{\prime}$ direction are shown in Figs. 2(a) and (b), taken at the same photon energies $\nu$ as Figs. 1. d) and (e), respectively. The two measurements show the states SS1+ and SS1- with different intensities, but otherwise at the same energy and having the same dispersion, confirming that they are of two-dimensional character. The different intensities are likely due to final state effects. There are small differences in binding energies between experiment and calculation on the order of $100 \mathrm{meV}$. One likely source of this discrepancy is surface relaxation which is neglected in the calculation. Constant energy contours obtained at the energies around the Dirac point, shown in Fig. 2(d) for the energies labelled in Fig. 2(a), clearly reveal the two band maxima in the $\mathrm{S}-\Gamma^{\prime}$ direction due to the strongly anisotropic Rashba splitting (see also Ref. 41).

Data and calculations yield a further set of surface states at higher binding energies, which we label SS2+ and SS2-. As opposed to the hole-like $\mathrm{SS} 1 \pm$ states, $\mathrm{SS} 2 \pm$ have an electron-like dispersion. In the experiment, they are most clearly resolved with $\nu=21.2 \mathrm{eV}$ (Fig. 2(a)). They are located near the bottom of the directional band gap at the S-point and quickly develop into surface resonances when moving away from $S$.

Besides the surface states found at the $S$ points, the calculations reveal an additional pair of surfaces states at the $\Gamma$ point (labelled by SSO \pm in Figs. $1(\mathrm{f})$ and $(\mathrm{g})$ ), which are in the unoccupied states and thus inaccessible to ARPES. For one termination, this state has been detected previously by STS 29. While the Dirac-cone states at the $S$ point are present even if SOC is neglected, the Dirac state at the $\Gamma$ point appears within a gap opened up by SOC and arises as a consequence of an SOC-driven band inversion. This scenario is reminiscent of the topological insulator $\mathrm{Bi}_{2} \mathrm{Se}_{3}[5$, indicating a possible topological origin. Here we show the signature of the surface state at $\Gamma$ for both terminations from tunneling spectra, see Fig. 3. The terminations in the STM data have been identified from the surface corrugation (compare fig. 3(a-c)). Spectra of the surface state (fig. 3(d) show only a very small shift of $\sim 6 \mathrm{meV}$ between the two terminations, with the surface state showing up at larger energies on the termination which we identify as the $(0 \overline{1} 0)$ surface.

We note that the band crossings of the Dirac states both at the $\Gamma$ and $S$ points are protected by time-reversal symmetry due to Kramers' theorem. Consistently for all surface states in the occupied states (SS1, \pm SS2 \pm ) those on the (010) surface occur at an energy at least $100 \mathrm{meV}$ higher than on $(0 \overline{1} 0)$, whereas the shift is very small and in the opposite direction for the surface state in the unoccupied states ( $\mathrm{SS} 0 \pm$ ).

We have fitted the standard Rashba-Bychkov 

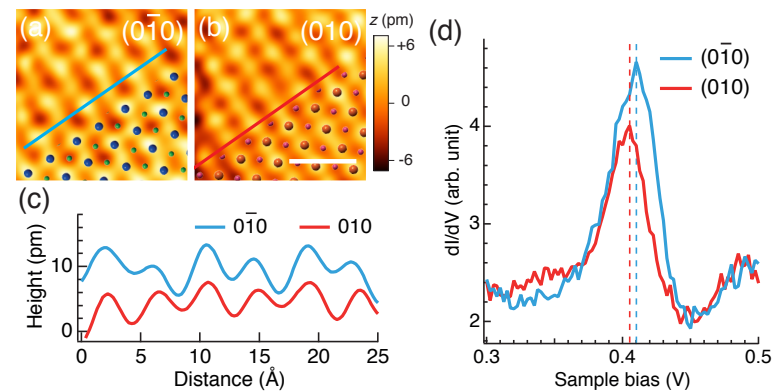

FIG. 3. (a) and (b) Topographies of the (010) and (010) terminations respectively, obtained with the same tip. Blue/red spheres represent $\mathrm{Bi}$ atoms and green/purple $\mathrm{Pd}$ atoms in the top surface layer, compare fig. 1(a). (c) Linecuts of the two terminations, showing the different corrugations. Linecuts shifted horizontally for clarity. (d) $d I / d V$ spectra obtained on $(0 \overline{1} 0)$ and (010) terminations. The surface state on the $(0 \overline{1} 0)$ face is at a slightly larger energy than on $(010)\left(V_{\mathrm{s}}=0.5 \mathrm{~V}\right.$, $\left.I_{\mathrm{s}}=2 \mathrm{nA}\right)$.

model [47 to cuts through the experimental band structure maps along the high-symmetry directions to extract the magnitude of spin splitting for the most prominent surface state, SS1+. The dispersion about the high-symmetry $S$ point is modeled as

$$
E_{ \pm}(k)=\frac{\hbar^{2}}{2 m^{*}}\left(|k| \pm k_{\mathrm{R}}\right)^{2}+E_{0},
$$

where $k$ denotes the momentum along the chosen direction in the surface BZ, $m^{*}$ is the effective mass, and $k_{\mathrm{R}}$ and $E_{0}$ denote the momentum offset and the energy of the band maxima, respectively. We quantify the size of the Rashba splitting by the momentum offset $k_{\mathrm{R}}$ and the energy difference $E_{\mathrm{R}}=\hbar^{2} k_{\mathrm{R}}^{2} /\left(2 m^{*}\right)$ between the band maximum $E_{0}$ and the band crossing point. The fits used to extract these parameters for SS1+ are shown in Figs. 4(a) and (b) for the $\mathrm{S}-\Gamma^{\prime}$ and $\mathrm{S}-\Gamma$ directions, respectively. The Rashba momentum offset $k_{\mathrm{R}}$ and energy $E_{\mathrm{R}}$ along the $\mathrm{S}-\Gamma^{\prime}$ direction in BiPd rank among the largest reported thus far, while both are significantly smaller in the $\mathrm{S}-\Gamma$ direction. The results are summarized and compared with a selection of previously reported values in Table [. Despite the large momentum offset, the Rashba parameter $\alpha_{\mathrm{R}}=\hbar^{2} k_{\mathrm{R}} / m^{*}$ of $\mathrm{BiPd}$ is smaller than for the $\mathrm{Bi} / \mathrm{Ag}(111)$ surface alloy due to the much larger effective mass of the surface states of BiPd. Large Rashba splittings, leading to well-separated spinsplit bands, may prove useful for applications involving the transport of spin rather than charge. Interesting, the Figure 4(c) shows a three-dimensional representation of the dispersion of SS1+ near the S-point, highlighting the anisotropy in the Rashba spin-splitting.

Since BiPd is noncentrosymmetric and no symmetry element can transform a (010) surface into a (010) surface, the shapes and energies of these surfaces' Dirac

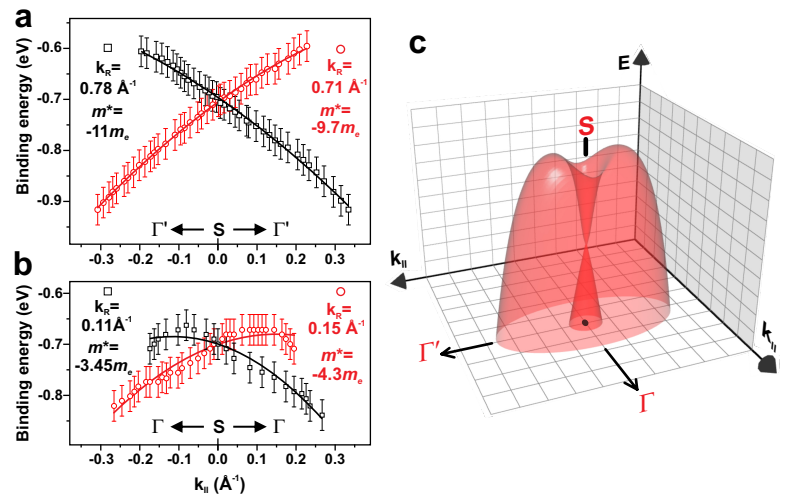

FIG. 4. (a) and (b) Cuts in the $\mathrm{S}-\Gamma^{\prime}$ and $\mathrm{S}-\Gamma$ directions, respectively, from which the band structure parameters of the SS1+ state have been determined. Solid lines show a fit of the Rashba model [Eq. (1)] to the data. (c) Band structure of the Rashba-spin split surface state at the S-point (SS1+) as determined by fitting the Rashba model to the ARPES data. The Rashba spin splitting is highly anisotropic.

TABLE I. Rashba momentum $k_{\mathrm{R}}$ in $\AA^{-1}$, Rashba energy $E_{\mathrm{R}}$ in meV, and Rashba parameter $\alpha_{\mathrm{R}}$ in $\mathrm{eV} \cdot \AA$ of a selection of materials with large Rashba-type band splitting.

\begin{tabular}{llclc}
\hline Sample & \multicolumn{1}{c}{$k_{\mathrm{R}}$} & $E_{\mathrm{R}}$ & $\alpha_{\mathrm{R}}$ & Ref. \\
\hline \hline $\mathrm{Au}(111)$ & 0.012 & 2.1 & 0.33 & {$[48$} \\
$\mathrm{Bi}(111)$ & 0.05 & 14 & 0.55 & $\underline{49}$ \\
$\mathrm{Bi} / \mathrm{Ag}$ surface alloy & 0.13 & 200 & 3.05 & {$[8$} \\
$\mathrm{BiTeI}$ & 0.052 & 100 & 3.8 & 12 \\
$\mathrm{BaNiS}$ & 0.2 & 150 & 0.26 & 50 \\
$\mathrm{Cs} / \mathrm{InSb}(110)$ & 0.0028 & 0.98 & 0.7 & 51 \\
$\mathrm{BiPd} \mathrm{SS1+,} \mathrm{S}-\Gamma$ & 0.13 & 17 & 0.25 & this work \\
$\mathrm{BiPd} \mathrm{SS} 1+, \mathrm{S}-\Gamma^{\prime}$ & 0.75 & 208 & 0.55 this work \\
\hline
\end{tabular}

states can be quite different, and indeed this is what we observe.

Our data reveal a surprising richness of Dirac surface states on the $(010) /(0 \overline{1} 0)$ surfaces of BiPd. Evidence for a surface state above $E_{\mathrm{F}}$ at $\Gamma 29$ and the observation of surface state SS1+ have been recently reported 39] (although with a different assignment of the $S$ and $\Gamma$ points in the latter). From a detailed comparison of calculations, ARPES and STM data we can identify two distinct surface states below the Fermi level at the $S$ point and one at the $\Gamma$ point, on each surface. The data reveal signatures of surface states from opposite orientations of the cystallographic $b$-axis, which occur on opposite faces of an ideal crystal, implying twinning on the scale of the ARPES spot size. Macroscopic studies of the impact of the lack of inversion symmetry on the material properties may therefore need to detwin the material to yield information from a single domain. The overall consistency of our results with the previously published data confirms the high reproducibility of the properties of BiPd.

The Rashba splitting of the surface states at the S- 
point exhibits a strong anisotropy, suggesting strongly directionally-dependent SOC in the surface state. This strong directional dependence can be understood by comparison with the surface structure of BiPd: the $\Gamma-\mathrm{S}$ direction is along rows of $\mathrm{Bi}$ (or $\mathrm{Pd}$ ) atoms, therefore electronic states propagating along this direction are only moderately exposed to the surface corrugation. Along the $\Gamma-\mathrm{S}^{\prime}$ (or equivalently $\mathrm{S}-\Gamma^{\prime}$ ) direction, rows of $\mathrm{Bi}$ and $\mathrm{Pd}$ atoms alternate, and electronic states with wavevectors along this direction are exposed much more strongly to the surface corrugation and hence to the surface electric fields which generate the spin splitting. The connection between surface corrugation and the spin-orbit splitting has been discussed previously in the context of the $\mathrm{Bi} / \mathrm{Ag}(111)$ surface alloy 52,53 . In BiPd, the corrugation of the top-most layer is a direct consequence of the crystal structure of the bulk material, boosting the spin splitting of the surface states only in specific directions due to the anisotropy of the crystal structure.

In summary, through comparison of ARPES and STM experiments with band structure calculations, we have confirmed the presence of unconventional Dirac surface states in noncentrosymmetric $\mathrm{BiPd}$, where the spinorbital texture on opposite faces is not simply related by symmetry. The extremely large and anisotropic Rashba splitting in this system makes it an excellent candidate for future studies on the intricate spin texture of spinsplit bands. Our results suggest a new way to engineer anisotropic spin textures and Rashba splittings of surface states by exploiting the low symmetry of the surface termination. The findings provide independent confirmation of the existence of twin boundaries in the material [29], which may prove crucial to understanding its superconducting properties [30, 54].

Acknowledgments. - The authors thank Ed Yelland for useful discussions. Funding from the MPG-UBC center and the Engineering and Physical Sciences Research Council (EP/I031014/1 and EP/L505079/1) are acknowledged. This work was supported by the DFG within projects STA315/8-1 and BE5190/1-1. We also thank the staff at Bessy II of the Helmholtz-Zentrum Berlin for their assistance.

* h.benia@fkf.mpg.de

†wahl@st-andrews.ac.uk

[1] M. Z. Hasan and C. L. Kane, Rev. Mod. Phys. 82, 3045 (2010)

[2] X.-L. Qi and S.-C. Zhang, Rev. Mod. Phys. 83, 1057 (2011).

[3] M. Z. Hasan and J. E. Moore, Ann. Rev. Cond. Matt. Phys. 2, 55 (2011)

[4] D. Hsieh, Y. Xia, L. Wray, D. Qian, A. Pal, J. H. Dil, J. Osterwalder, F. Meier, G. Bihlmayer, C. L. Kane, Y. S. Hor, R. J. Cava, and M. Z. Hasan, Science 323, 919
$(2009)$

[5] Y. Xia, D. Qian, D. Hsieh, L. Wray, A. Pal, H. Lin, A. Bansil, D. Grauer, Y. S. Hor, R. J. Cava, and M. Z. Hasan, Nat Phys 5, 398 (2009).

[6] J. Seo, P. Roushan, H. Beidenkopf, Y. S. Hor, R. J. Cava, and A. Yazdani, Nature 466, 343 (2010)

[7] E. I. Rashba, Sov. Phys. Solid State 2, 1109 (1960).

[8] C. R. Ast, J. Henk, A. Ernst, L. Moreschini, M. C. Falub, D. Pacilé, P. Bruno, K. Kern, and M. Grioni, Phys. Rev. Lett. 98, 186807 (2007).

[9] A. Crepaldi, L. Moreschini, G. Autès, C. TournierColletta, S. Moser, N. Virk, H. Berger, P. Bugnon, Y. J. Chang, K. Kern, A. Bostwick, E. Rotenberg, O. V. Yazyev, and M. Grioni, Phys. Rev. Lett. 109, 096803 (2012)

[10] M. Bahramy, B.-J. Yang, R. Arita, and N. Nagaosa, Nat. Commun. 3, 679 (2012)

11] D. Hsieh, Y. Xia, D. Qian, L. Wray, J. H. Dil, F. Meier, J. Osterwalder, L. Patthey, J. G. Checkelsky, N. P. Ong, A. V. Fedorov, H. Lin, A. Bansil, D. Grauer, Y. S. Hor, R. J. Cava, and M. Z. Hasan, Nature 460, 1101 (2009).

[12] K. Ishizaka, M. S. Bahramy, H. Murakawa, M. Sakano, T. Shimojima, T. Sonobe, K. Koizumi, S. Shin, H. Miyahara, A. Kimura, K. Miyamoto, T. Okuda, H. Namatame, M. Taniguchi, R. Arita, N. Nagaosa, K. Kobayashi, Y. Murakami, R. Kumai, Y. Kaneko, Y. Onose, and Y. Tokura, Nat. Mater. 10, 521 (2011).

[13] Y. H. Wang, D. Hsieh, D. Pilon, L. Fu, D. R. Gardner, Y. S. Lee, and N. Gedik, Phys. Rev. Lett. 107, 207602 (2011).

[14] E. Bauer and M. Sigrist, Non-Centrosymmetric Superconductors: Introduction and Overview, Lecture Notes in Physics, Vol. 847 (Springer Berlin, 2012) pp. 1-357.

[15] E. Bauer, G. Hilscher, H. Michor, C. Paul, E. W. Scheidt, A. Gribanov, Y. Seropegin, H. Noël, M. Sigrist, and P. Rogl, Phys. Rev. Lett. 92, 027003 (2004).

[16] A. P. Schnyder, S. Ryu, A. Furusaki, and A. W. W. Ludwig, Phys. Rev. B 78, 195125 (2008)

[17] B. Béri, Phys. Rev. B 81, 134515 (2010)

[18] A. P. Schnyder and S. Ryu, Phys. Rev. B 84, 060504 (2011)

[19] M. Sato and S. Fujimoto, Phys. Rev. B 79, 094504 (2009)

[20] D. M. Kheiker, G. S. Zhdanov, and N. N. Zhuravlev, Zh. Eksp. Teor. Fiz. 25, 621 (1953).

[21] N. Zhuravlev, Zh. Eksp. Teor. Fiz. 5, 1064 (1957).

[22] Y. Bhatt and K. Schubert, Journal of the Less Common Metals 70, P39 (1980).

[23] Y. Bhatt and K. Schubert, J. Less-Common Met. 64, P17 (1979)

[24] V. M. Ionov, N. A. Tomilin, A. E. Prozorovskii, A. N. Klimenko, Y. V. Titov, S. G. Zhukov, and G. V. Fetisov, Sov. Phys. Crystallogr. 34, 496 (1989).

[25] N. E. Alekseevskii, Zh. Eksp. Teor. Fiz. 23, 484 (1952).

[26] B. Joshi, A. Thamizhavel, and S. Ramakrishnan, Phys. Rev. B 84, 064518 (2011).

[27] M. Mondal, B. Joshi, S. Kumar, A. Kamlapure, S. C. Ganguli, A. Thamizhavel, S. S. Mandal, S. Ramakrishnan, and P. Raychaudhuri, Phys. Rev. B 86, 094520 (2012)

[28] K. Matano, S. Maeda, H. Sawaoka, Y. Muro, T. Takabatake, B. Joshi, S. Ramakrishnan, K. Kawashima, J. Akimitsu, and G.-q. Zheng, J. Phys. Soc. Jpn. 82, 084711 (2013)

[29] Z. Sun, M. Enayat, A. Maldonado, C. Lithgow, E. Yel- 
land, D. C. Peets, A. Yaresko, A. P. Schnyder, and P. Wahl, Nat Commun 6, 6633 (2015).

[30] D. C. Peets, A. Maldonado, M. Enayat, Z. Sun, P. Wahl, and A. P. Schnyder, Phys. Rev. B 93, 174504 (2016)

[31] S. Sasaki, M. Kriener, K. Segawa, K. Yada, Y. Tanaka, M. Sato, and Y. Ando, Phys. Rev. Lett. 107, 217001 (2011)

[32] N. Levy, T. Zhang, J. Ha, F. Sharifi, A. A. Talin, Y. Kuk, and J. A. Stroscio, Phys. Rev. Lett. 110, 117001 (2013)

[33] C. Liu, Y. Lee, T. Kondo, E. D. Mun, M. Caudle, B. N. Harmon, S. L. Budko, P. C. Canfield, and A. Kaminski, Phys. Rev. B 83, 205133 (2011)

[34] H. Kim, K. Wang, Y. Nakajima, R. Hu, S. Ziemak, P. Syers, L. Wang, H. Hodovanets, J. D. Denlinger, P. M. R. Brydon, D. F. Agterberg, M. A. Tanatar, R. Prozorov, and J. Paglione, "Beyond spin-triplet: Nodal topological superconductivity in a noncentrosymmetric semimetal," (2016), arXiv:1603.03375 [condmat.supr-con]

[35] Y. Nakajima, R. Hu, K. Kirshenbaum, A. Hughes, P. Syers, X. Wang, K. Wang, R. Wang, S. R. Saha, D. Pratt, J. W. Lynn, and J. Paglione, Sci. Adv. 1, e1500242 (2016)

[36] M. N. Ali, Q. D. Gibson, T. Klimczuk, and R. J. Cava, Phys. Rev. B 89, 020505 (2014)

[37] G. Bian, T.-R. Chang, R. Sankar, S.-Y. Xu, H. Zheng, T. Neupert, C.-K. Chiu, S.-M. Huang, G. Chang, I. Belopolski, D. S. Sanchez, M. Neupane, N. Alidoust, C. Liu, B. Wang, C.-C. Lee, H.-T. Jeng, C. Zhang, Z. Yuan, S. Jia, A. Bansil, F. Chou, H. Lin, and M. Z. Hasan, Nat. Commun. 7, 10556 (2016).

[38] S.-Y. Guan, P.-J. Chen, M.-W. Chu, R. Sankar, F. Chou, H.-T. Jeng, C.-S. Chang, and T.-M. Chuang, "Superconducting topological surface states in non-centrosymmetric bulk superconductor $\mathrm{PbTaSe}_{2}$," (2016), arXiv:1605.00548 [cond-mat.supr-con].

[39] M. Neupane, N. Alidoust, S.-Y. Xu, I. Belopolski, D. S. Sanchez, T.-R. Chang, H.-T. Jeng, H. Lin, A. Bansil, D. Kaczorowski, M. Z. Hasan, and T. Durakiewicz, "Discovery of the topological surface state in a noncentrosym- metric superconductor BiPd," (2015), arXiv:1505.03466 [cond-mat.mes-hall].

[40] D. C. Peets, J. Phys.: Conf. Ser. 568, 022037 (2014).

[41] See Supplemental Material at http://link.aps.org/ supplemental/10.1103/... for additional ARPES measurements.

[42] S. C. White, U. R. Singh, and P. Wahl, Review of Scientific Instruments 82, 113708 (2011)

[43] O. K. Andersen, Phys. Rev. B 12, 3060 (1975)

[44] V. Antonov, B. Harmon, and A. Yaresko, Electronic structure and magneto-optical properties of solids (Kluwer Academic Publishers, Dordrecht, Boston, London, 2004).

[45] A. Y. Perlov, A. N. Yaresko, and V. N. Antonov, PYLMTO: A Spin-Polarized Relativistic LMTO Package for Electronic Structure Calculations (unpublished).

[46] A. H. Macdonald, W. E. Pickett, and D. D. Koelling, J. Phys. C 13, 2675 (1980).

[47] Y. A. Bychkov and É. I. Rashba, JETP Lett. 39, 78 (1984)

[48] S. LaShell, B. A. McDougall, and E. Jensen, Phys. Rev. Lett. 77, 3419 (1996)

[49] Y. M. Koroteev, G. Bihlmayer, J. E. Gayone, E. V. Chulkov, S. Blügel, P. M. Echenique, and P. Hofmann, Phys. Rev. Lett. 93, 046403 (2004).

[50] D. Santos-Cottin, M. Casula, G. Lantz, Y. Klein, L. Petaccia, P. Le Fèvre, F. Bertran, E. Papalazarou, M. Marsi, and A. Gauzzi, Nat. Commun. 7, 11258 (2016)

[51] J. R. Bindel, M. Pezzotta, J. Ulrich, M. Liebmann, E. Y. Sherman, and M. Morgenstern, Nature Physics (2016), 10.1038/nphys3774, advance online publication.

[52] I. Gierz, B. Stadtmüller, J. Vuorinen, M. Lindroos, F. Meier, J. H. Dil, K. Kern, and C. R. Ast, Physical Review B 81, 245430 (2010)

[53] G. Bian, X. Wang, T. Miller, and T.-C. Chiang, Physical Review B 88, 085427 (2013)

[54] X. B. Yan, Y. Xu, L. P. He, J. K. Dong, H. B. Cho, D. C. Peets, J.-G. Park, and S. Y. Li, Supercond. Sci. Tech. 29, 065001 (2016). 